\title{
A modified tabu search algorithm for the single-machine scheduling problem using additive manufacturing technology
}

\author{
Marcello Fera $^{a^{*}}$, Roberto Macchiaroli ${ }^{a}$, Fabio Fruggiero ${ }^{b}$, and Alfredo Lambiase ${ }^{c}$
}

${ }^{a}$ Department of Engineering, University of Campania "Luigi Vanvitelli", Via Roma 29, Aversa (CE), Italy

${ }^{b}$ University of Basilicata, School of Engineering, Via Nazario Sauro, 85, Potenza, PZ, Italy

${ }^{c}$ Department of Industrial Engineering, University of Salerno, Via Giovanni Paolo II, Fisciano, SA, Italy

\section{CH R O N I C L E}

Article history:

Received October 82019

Received in Revised Format

December 282019

Accepted December 312019

Available online

January 22020

Keywords:

Additive Manufacturing

Scheduling

Heuristics

Production Planning

\begin{abstract}
A B S T R A C T
The Additive Manufacturing (AM) scheduling problem is becoming a very felt issue not only by the scholars but also by the practitioners who are looking to this new technology as a new integrated part of their traditional production systems. They need new scheduling models to adapt the traditional scheduling rules to the changed ones of the additive manufacturing. This paper deals with the enhancement of a scheduling problem for additive manufacturing just present in literature and the presentation of a new meta-heursitic (adapted to the new requirements of the additive manufacturing technology) based on the tabu-search algorithms.
\end{abstract}

(C) 2020 by the authors; licensee Growing Science, Canada

\section{Introduction}

From the beginning of the research works on the AM, the issues related to the operations management were not put in great evidence because the main goal for the starting research was to demonstrate the quality and the mechanical capability of parts realised with this new technology (Fera et al., 2016). This research effort was paid more and more in almost 30 years of research. Nevertheless, the operational issues related to the use of AM machines became a urgent issue to be faced when this kind of technology arrived in the real production contexts and the industrial practitioners called for new methodologies able to face the different rules of this new technology. These operational issues were faced in last ten years of research starting from the measurement of the cost performances of this technology (Ruffo \& Hague, 2007; Atzeni \& Salmi, 2012; Rickenbacher et al., 2013, Costabile et al., 2017; Fera et al., 2017, 2018), arriving to the supplying problems related to the AM machines (Strong et al., 2018; Khajavi et al., 2014; Verboeket \& Krikke, 2019). In this paper we will focus on another operations management issue that is the scheduling of these kind of machines and in particular we will investigate a solution method to improve the reduction of calculation time and the quality of the solutions. This aim will be achieved starting from a mathematical model just present in literature, i.e. (Fera et al., 2018). To this mathematical

* Corresponding author

E-mail: marcello.fera@unicampania.it (M. Fera)

2020 Growing Science Ltd.

doi: $10.5267 /$ j.ijiec.2020.1.001 
model some parts will be added to make it more efficient; afterwards a modification of the traditional tabu-search algorithm will be presented and then compared to the meta-heuristic used in (Fera et al., 2018) that is the genetic algorithm, to understand which are the advantages and disadvantages in using one or another to solve the scheduling problem cited.. In section 2 it is provided a wide literature review about AM production planning and scheduling. In $3^{\text {rd }}$ section, the starting mathematical representation of additive manufacturing scheduling problem (AMSP) is re-proposed adding some specifications and corrections to the model just presented in Fera et al. (2018). Section 4 provides the modification of the tabu-search algorithm and in the section 5 the modified algorithm will be compared in terms of results to the one used in (Fera et al., 2018). Finally, conclusions are drawn in Section 6, the results from the test case are discussed and future research and improvements are presented.

\section{Literature review}

As before anticipated, the AM research started more or less thirty years ago and during this time span several engineering research issues were faced. According to Witherell et al. (2017) the AM research topics are associated with many fields of the engineering knowledge and, in particular, they are related to the design of product and process engineering. Moreover, as far as management issues are concerned, AM was studied from several viewpoints. For instance, in Pour et al. (2016) the problem is faced through a reconfiguration of production systems and supply chains, and the use of this kind of technology is individuated as a main source of improvement for both systems. Fruggiero at al. (2016) studied interactions between humans and machines using AM technology. It is probably also worth to note that AM is generally listed as a key enabling technology for the achievement of the Industry 4.0 paradigm (Dilberoglu et al., 2017; Fruggiero et al., 2016).

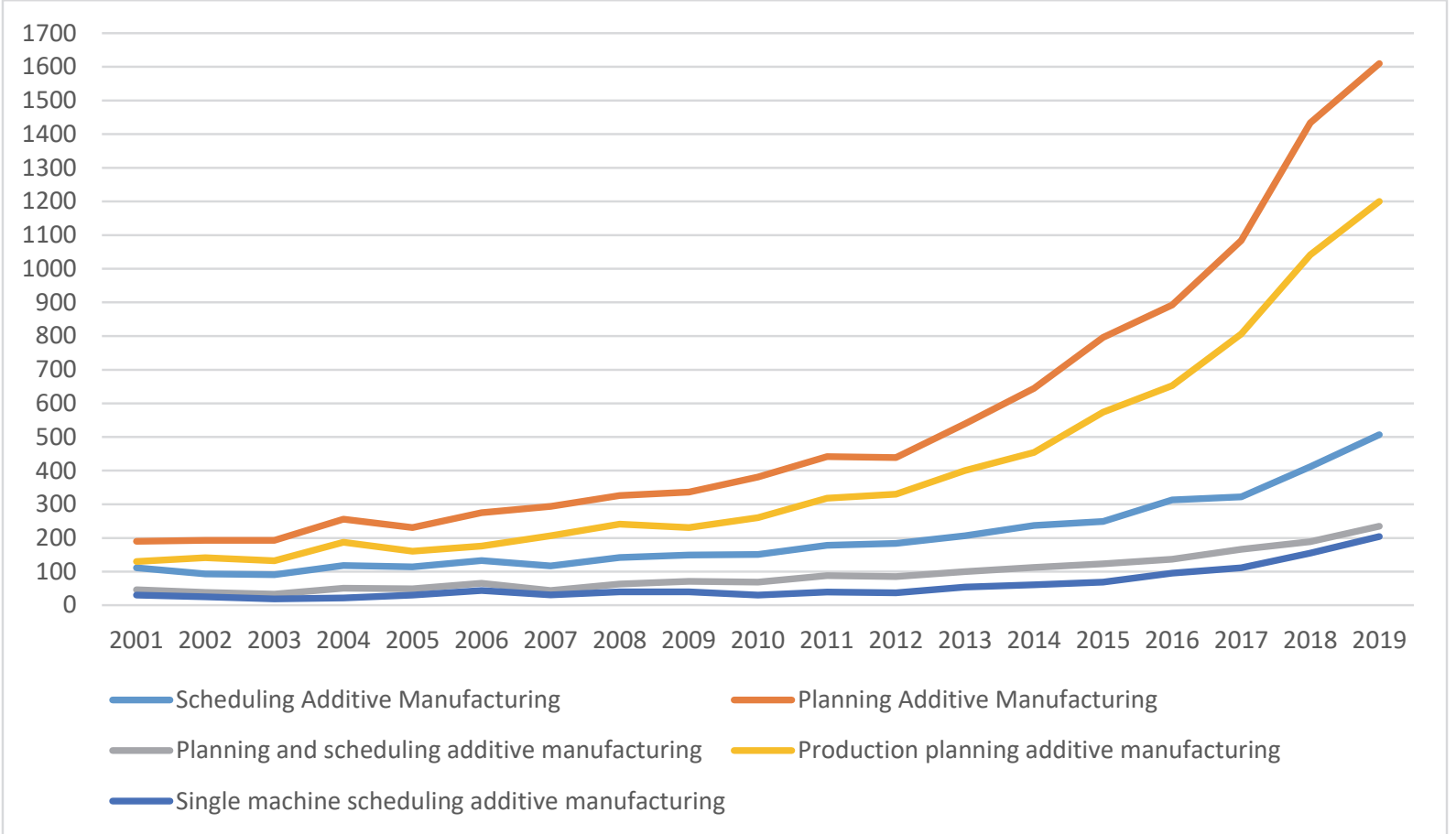

Fig. 1. The growth of interest for the research themes related to the additive manufacturing operations scheduling and planning

Since this technology is recognised as a mature technology to be inserted in the value flow of industries, it needs to be analysed in terms of planning and scheduling to allow their management in conjunction 
with all the other machines of the production environment and objective of this paragraph will be focused on the study of the actual state of the art about the scheduling models for single machines using AM technology. To this extent, one of the main research databases today available will be inquired to understand how much the aim of this paper was studied in the past and what is the state of the art about the planning and scheduling for AM in general. The chosen database for the systematic literature review is ScienceDirect ${ }^{\mathbb{B}}$. The research words used are: (i) scheduling additive manufacturing, (ii) planning additive manufacturing, (iii) planning and scheduling additive manufacturing, (iv) production planning additive manufacturing and (v) single machine scheduling additive manufacturing. At a first attempt, the search on the database previously indicated showed more than thirty thousand papers available, from 2001 to date. For all the research themes indicated, the trend of the number of papers WAs growing during the time as shown in Fig. 1. Once it was cleared that the interest about the themes previously indicated, it was investigated which are the journals that give more attention to these research themes, in the following graph it is shown the number of papers for each journal individuated between the most popular on this theme.

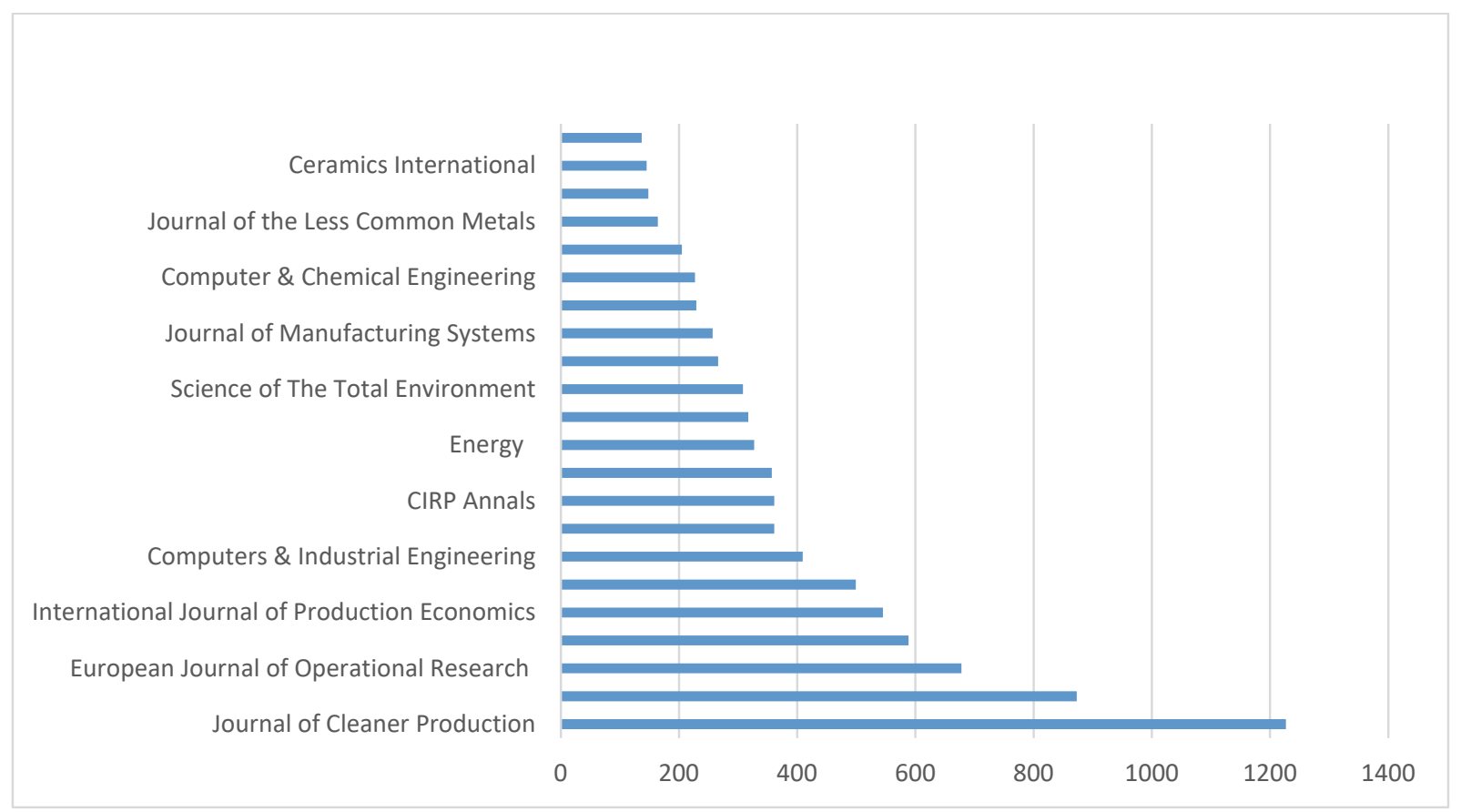

Fig. 2. The number of papers on planning and scheduling for each journals

As it is evident from Fig. 2, the main journal that publishes researches about the planning and scheduling of AM is Journal of Cleaner Production edited by Elsevier, since this forum is dedicated for the sustainability of the productions. The second and third ones are close to the classical world of the operations management, respectively. The first more concentrated on the mathematical aspects of the scheduling problem, the second more focused on the management of operations issues. The same of this last case can be said on fourth and fifth journal of Fig. 2. Within the first five journals, that covers almost the $50 \%$ of the total number of papers about the planning and scheduling for AM, the research themes investigated are divided as shown in Fig. 3. As it is clear from Fig. 3 the main interests and the main results of research are in the field of planning and scheduling even if most part of the paper selected, using the method here presented, are not strictly related to the AM issues. Moreover, the problem of single machine scheduling for AM is the less developed in the research world and also for this specific field of knowledge it is confirmed the fact that the problem faced are not related to the AM specific issues 
and rules. This result (in Fig. 4) is also confirmed by the analysis of the keywords of all the papers in the main journals before introduced on the specific research theme of the single machine scheduling.

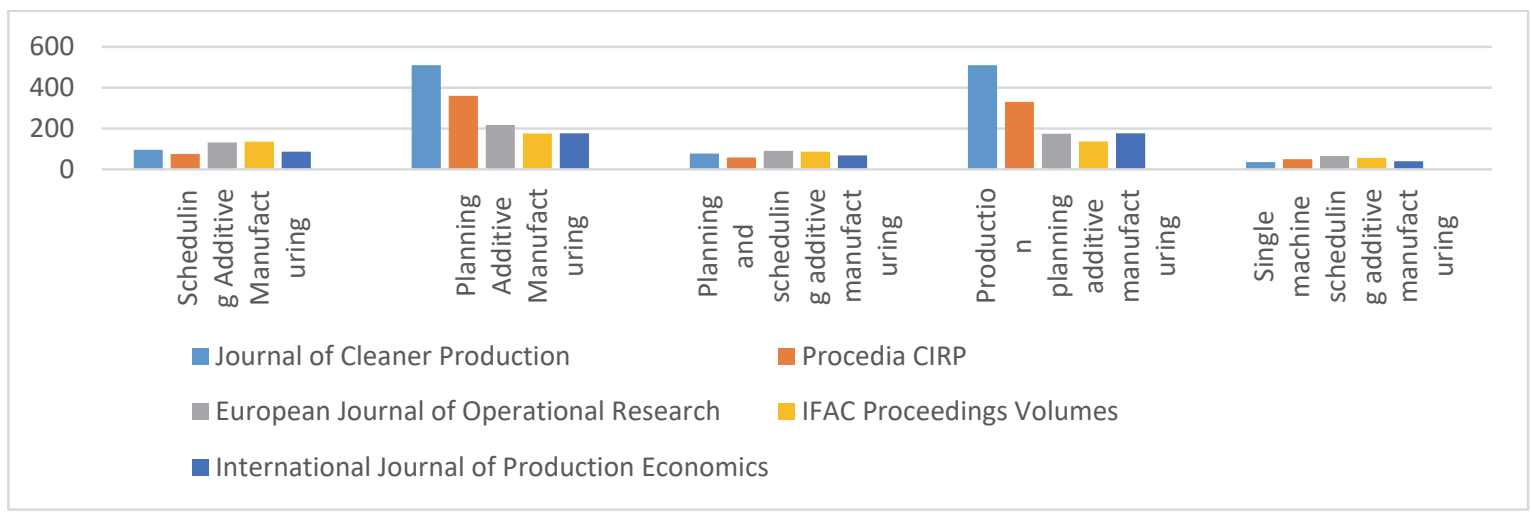

Fig. 3. The number of papers for each research theme for the main journals of interest

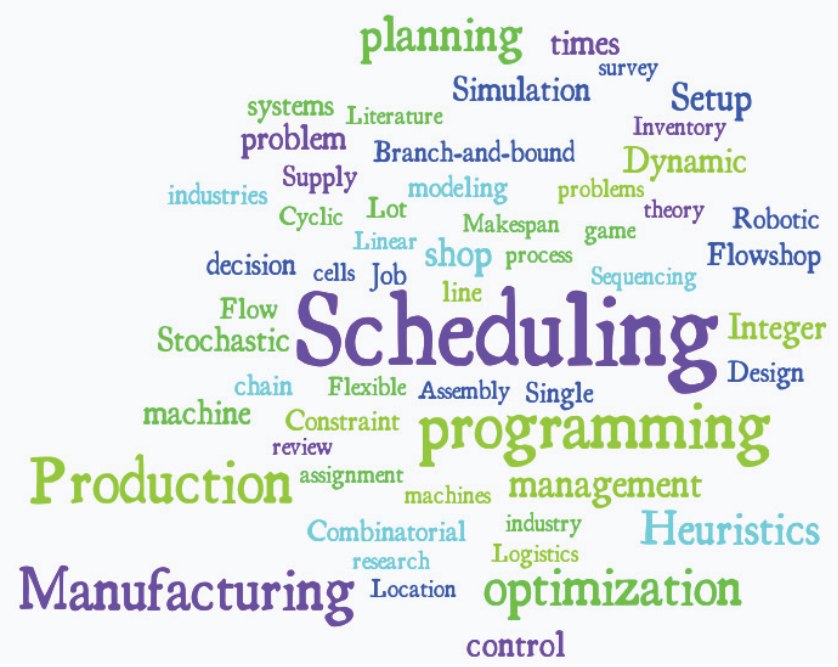

Fig. 4. The words cloud for single machine scheduling problem for AM in the five most important journals individuated

The keywords were analysed using the word cloud method that reports the words more frequent in a database with different size of the word to represent the growing frequency. In Fig. 4, 322 keywords were analysed and only the words with a higher word count than three is reported. As it is easy to see from the Fig. 4 the word manufacturing is present in many sources, but the word additive is not, this is mainly due to the fact that models developed are not strictly related to the problem of additive manufacturing. Starting from this point, the aim of this paper is to focus on the attention on this weak research field. Following in this paragraph, a specific literature review on the few models about the single machine scheduling problem for AM is presented. The first time in which the process planning using AM appeared as a main research issue was in 2008 (Ren et al., 2008) but it was not deeply investigated until a mechanically reliable metal was available. Li et al. (2017) proposed work about distributed AM machines' production planning when the machines are called to realise a cumulative demand created by the sum of several different small quantity orders by also different clients; the authors, recognising the problem as NP-HARD, defined several heuristics on the geometrical constraints principle when an AM machine was used. Therefore, the study by Li et al. (2017) could be of reference for our purpose but it refers to the raw-material cost minimisation that is not useful for the aim of the production flow 
optimisation. Ransikarbum et al. (2017) proposed a decision-support model based on the orientation optimisation of a unique PN on multiple AM machines. This model refers to a multi-machine environment and considers the possibility to produce only one-part type at the time; it does not exactly match the objective of this paper. Moreover, it uses an optimisation process based on the part orientation, which is out of the scope herein that wants to optimise the E\&T optimising the lot sizing of the single build run. Jin et al. (2017) focused on the process planning theme using AM. Additionally, this research deals with the definition of process planning to minimise the raw-material consumption for the AM; therefore, for the same reasons of the paper by Li et al. (2017), it is possible to say that the model does not fit exactly with the aim of the optimisation as it is intended in this paper. Another interesting work on the assignment of a specific job to a build was presented by Zhang et al. (2016), who focused on the optimal multiple parts placing in the building chamber with respect to user-defined objectives but it is very far from the E\&T minimisation problem for the single-machine scheduling that we want to face. $\mathrm{Zu}$ et al. (2017) based their research on a framework defined by Newman et al. (2015) and they investigated specifically the combination of the old and new production technologies in terms of process planning with an objective to optimise the modification process of existing products. In 2018, Chergui et al. developed a model capable to schedule the production to satisfy a certain demand level, minimizing the tardiness of the orders; the problem is solved applying a heuristic written in Python. Recently some researchers more focused on the AM different production organizations such as the single machine, parallel identical machines and non-identical ones faced the problem of the scheduling also. Kucukkoc in 2019 developed a research about the comparison of these three different production organizations identifying a mathematical formulation of this problem with the objective function focused on the makespan and applying the algorithms of CPLEX by IBM to solve the problems (Kucukkoc, 2019). In 2018 Fera et al. developed a mathematical model that tries to optimize a multi-objective function based on the earliness \& tardiness $(\mathrm{E} \& \mathrm{~T})$ and on the production cost subject to several constraints related to the demand satisfaction and to the respect of the chamber volume of the AM single machine. The problem was solved using a modified genetic algorithm capable of elaborating the specific rules of management imposed by the AM. As it is clear from the previous papers, the single machine scheduling problem applied to the AM, from a cost and time point of view, is faced only by Fera et al. (2018). For this reason, here a modification of the original mathematical model and a modified tabu search algorithm will be presented to be compared with the genetic algorithm that was used in the original paper.

\section{The proposed scheduling model}

As introduced earlier, this paper deals with the presentation of some modifications of the model presented in Fera et al. (2018) and of a new heuristic result when applied to the mathematical model here presented. It is worth to note that the tabu-search here presented needed to be changed from its traditional formulation due to the different management needs of the AM. The following modifications are implemented in the past model:

(i) the weights for the E\&T are no more defined by mathematical formulas but left to the decision maker (i.e. the decision maker will decide autonomously if the earliness is more important than the tardiness, the contrary or they are equally important for his application case) and

(ii) the completion time of the generic order for a specific part number is now defined in detail.

The model to optimise the production scheduling has a double objective, the completion time $\left(C T_{i}\right)$ and total order cost $\left(T O C_{i}\right)$. Given these definitions, let us to report the mathematical formulation of the optimisation problem analysed by Fera et al. (2018) that is a NP-HARD problem. 


$$
F_{S}=F_{E T}+F_{C P}
$$

where

$$
\begin{aligned}
& F_{E T} \\
& =\sum_{i=1}^{n_{g}}\left[\begin{array}{lll}
\alpha & E_{i}+\beta & T_{i}
\end{array}\right] \\
& F_{C P}=\sum_{i=1}^{n_{g}} \gamma_{S} T O C_{i} \\
& \sum_{i=1}^{n g} n_{i, j} * V_{i} \leq V_{\text {chamber }} \forall j \in\left[1, n_{b}\right] \\
& \sum_{j=1}^{n b} n_{i, j}=d_{i}, \forall i \in\left[1, n_{g}\right] \\
& \alpha_{S}, \beta_{S}, \gamma_{S}, \text { TOC }_{i}, V_{i}, V_{\text {chamber }} \in \mathbb{R}^{+} \\
& E_{i}, T_{i}, i, j, n_{g}, n_{b} \in \mathbb{Z}^{+}
\end{aligned}
$$

where

$\begin{array}{llc}\alpha_{S}: & \text { Earliness constant weight } & {[1 / \text { day }]} \\ \beta_{S}: & \text { Tardiness constant weight } & {[1 / \text { day }]} \\ E_{i}: & \text { Earliness of the } i-\text { th geometry } & {[\text { day }]} \\ T_{i}: & \text { Tardiness of the } i-\text { th geometry } & {[\text { day }]} \\ \text { TOC }: \text { : } & \text { Total order cost of the } i-t h & {[€]} \\ & \text { geometry } & \\ \gamma_{S}: & \text { Cost constant weight } & {[1 / €]} \\ n_{g}: & \text { number of order/geometries } & {[-]} \\ n_{i, j} & \text { Number of the } i-\text { th item in } j-t h & {[\text { part }]} \\ & \text { build } & \\ V_{i} & \text { Volume of the } i-t h \text { geometry } & {\left[\mathrm{cm}^{3}\right]} \\ V_{c h a m b e r} & \text { Build chamber volume } & {\left[\mathrm{cm}^{3}\right]} \\ n_{b} & \text { Number of builds in the schedule } & {[-]} \\ d_{i}: & \text { demand of the } G_{i}-\text { th geometry } & {[\text { part }]}\end{array}$

So, the first issue to be solved by the production planner is the balance of E\&T (E\&T), which are traditionally defined for the $h$-th job as follows:

where

$$
E_{h}=\max \left(0, d d_{h}-C_{h}\right), T_{h}=\max \left(C_{h}-d d_{h}, 0\right),
$$

$$
\begin{array}{lll}
d d_{h}: & \text { Due date of the } h \text { - } t h \text { job } & {[\text { day }]} \\
C_{h}: & \text { Completion time of the } & {[\text { day }]} \\
& h \text {-th } \text { order }
\end{array}
$$

The common and traditional notation used to present the single-machine E\&T problem is 


$$
1 \| \sum_{h=1}^{n} \alpha E_{h}+\beta T_{h}
$$

where

$\begin{array}{lll}E_{h}: & \begin{array}{l}\text { Earliness of } h-t h \\ \text { job }\end{array} & {[\text { day }]} \\ T_{h}: & \begin{array}{l}\text { Tardiness of } h-t h \\ \text { job }\end{array} & {[\text { day }]} \\ n & \text { number of jobs } & {[\#]} \\ \alpha: & \text { constant weigh for } & {[1 / \text { day }]} \\ & \text { E } & \\ \beta: & \text { constant weigh for } & {[1 / \text { day }]}\end{array}$

As previously introduced, in the 2018 model the weights of E\&T, as obtained by the calculations of mathematical formulas, are considered but their values are in the decision field of the production manager that will consider more relevant the earliness or the tardiness according to strategic considerations, only respecting the fact that the E\&T weights complement each other. Lastly, in the paper the way to compute the completion time, needed for the calculation of earliness and tardiness reported previously, is presented.

$$
C_{h}=\sum_{j=1}^{n_{b^{*}}} t_{j} \text { where } \sum_{j=1}^{n_{b^{*}}} n_{h j}=d_{i} \forall h=1, n_{g}
$$

where

$$
\begin{array}{lll}
t_{j}: & \text { Building time for } j-t h \text { build } \\
n_{h j} & \begin{array}{l}
\text { number of units of the h-th geometry in } \\
\text { build } j
\end{array} \\
d_{h} & \begin{array}{l}
\text { Demand for geometry } i \\
n_{b} *
\end{array} & \begin{array}{l}
\text { Latest build in which geometry } i \text { is } \\
\text { produced }
\end{array} \\
n_{g} & \text { Number of geometries or orders }
\end{array}
$$

In any case, note the production time $t_{j}$ of each build depends on the type of parts that compose of the build. In fact, in the previous equation, it is considered that the time to complete at the last build $n b^{*}$ the total number of parts produced for each $h$-th part is equal to its demand $d_{i}$ is as expressed in the previous notation. Thanks to these two simple added formulas hopefully the mathematical model to optimize the scheduling of the AM machine will be easier and clear to be implemented in real cases.

\section{Solution methods}

As introduced in the previous paragraph of this paper a modification of the classical tabu-search (TS) algorithm will be presented to fit the specific issues related to the use of the AM machine technology. This method (i.e. TS) was originally introduced by Glover and Laguna (1997a,b); it is one of the most famous metaheuristic algorithms and is commonly used to solve continuous or discrete problems. The TS approach is simple but extremely efficient; it is a local search algorithm, which includes a mechanism to escape from the local minimum and to overcome the limitations of some common heuristics. TS needs an initial feasible solution to start exploration. Applying a move to the initial configuration, TS can 
produce new schedules but not all candidate solutions are acceptable. In fact, TS uses a memory structure, known as a Tabu list (TL), to avoid already analysed solutions. TL holds the current OF value $\left(f_{0}\right)$ and the attributes, i.e. all necessary information concerning performed moves. Each move produces a new value for the OF $\left(f_{\text {new }}\right)$ and, if it is better than the current one, the move is accepted, and the value of the OF is updated $\left(f_{0}=f_{\text {new }}\right)$. Once the TL is updated, with attributes and OF value, research is iterated as reported in Fig. 4. Moreover, it is possible to define specific search criteria, which introduce reinforcement towards some search directions and/or penalties towards others. TS has no convergence criterion but a termination criterion, such as in the example of maximum run time and number of iterations and unsuccessful (not improving) iterations. To understand how the TS algorithm will be specialised for the single AM scheduling problem, let us first give an example of how a move is applied to a standard single-machine scheduling problem. In a single-machine scheduling problem, there is a typical move, known as swap. Given a feasible job sequence on a machine, TS performs a swap of jobs in various ways:

- Following job swap

- Random job swap

These kinds of swap are hardly applicable to the AM scheduling problem (that has a matrix schema); therefore, it is necessary to give a definition of what a swap (or move) is for an AM scheduling problem. Swapping matrix columns (i.e. assigning the partial and total quantities of one geometry to another) is not a solution because total quantities to be produced for different geometries are, normally, different. Refer to the quantities of orders \#1 and \#2, highlighted in red, in Fig. 5.

\begin{tabular}{|c|c|c|c|c|c|c|}
\hline \multirow{2}{*}{\multicolumn{2}{|c|}{$S$}} & \multicolumn{5}{|c|}{ Geometries } \\
\hline & & 1 & 2 & 3 & $\ldots$ & $n_{g}$ \\
\hline \multirow{5}{*}{$\stackrel{\bar{\Xi}}{\bar{\Xi}}$} & 1 & $n_{1,1}$ & $n_{2,1}$ & $n_{31}$ & $\ldots$ & $n_{g / 1}$ \\
\hline & 2 & $n_{1,2}$ & $n_{2,2}$ & $n_{3,2}$ & $\ldots$ & $n_{g, 2}$ \\
\hline & 3 & $n_{1,3}$ & $n_{2,3}$ & $n_{3,3}$ & $\ldots$ & $n_{g, 3}$ \\
\hline & $\ldots$ & $\ldots$ & $\ldots$ & $\ldots$ & $\ldots$ & $\ldots$ \\
\hline & $n_{b}$ & $n_{l, n b}$ & $n_{2, n b}$ & $n_{3, n b}$ & $\ldots$ & $n_{n g, n b}$ \\
\hline
\end{tabular}

Fig. 5. Swap attempts

A second attempt could be to swap matrix rows, which represent builds. This move is possible because it comprises a build sequence exchange, without alteration of volume or production constraints, as we can see for \#1 and \#2 builds in Fig. 5. It is important to note that a full analysis of all possible row swaps is a very difficult objective, especially in case of a high number of builds, since the number of possible combinations is $n_{b}$ ! Nevertheless, the first experimental campaign revealed that build swapping produced very small improvements in the OF value. This might be due to the fact that, generally, the initial solution was already oriented to a great saturation of the production chamber. So, a simple swap between builds was not able to achieve significant improvements in terms of costs. However, this circumstance needs to be better investigated. However, this preliminary result shows that different swap moves had to be identified for the AM scheduling problem.-Before we introduce the new swap move, let us introduce the general TS algorithm applied in this paper (PTS), which specialises in a particular swap movement.-The first step of the algorithm is the definition of a first feasible solution, namely $S_{0}$, for which it would be possible to compute the corresponding value of the OF, namely $f_{0}$. After this first step, a random value for a parameter useful to change the elements of the first solution in the proposed swap move is decided; this parameter is namely $\varepsilon$, a positive integer, that will help to modify the quantity of the $j-t h$ build for the $i-t h$ geometry. After these two initialisation steps, the algorithm starts to investigate the local 
dominium of research applying small moves (compliant with the rule later defined) of the quantities. After executing the first local research, the algorithm finds the best value of the OF and associates it with the assignment solution, thus defining a new solution $S_{1}$; this becomes the new starting solution. The old one is registered in the TL, and the procedure is repeated. If in comparison with the OFs, the value of the new ones is equal to or less than the previous one, then a calculation module, named Differentiate Module, is executed to try to go out of the local minimum; if this attempt fails two times, the algorithm stops itself. The TS algorithm resumes before it reaches an organisation quite similar to the traditional TS. Moreover, it is quite clear that the Schedule Screening module is the main block of PTS and requires to be analysed deeply. In this module, the variation of the traditional swap model is present; the proposed swap moves a discrete quantity (indicated as $\varepsilon$,) between two different builds, which is randomly in a specified interval. The steps to apply this swap move are as follows:

- Set randomly a column $i$ in the matrix: $-i \in\left[1, n_{g}\right]$;

- Set randomly first build, called picking build: $b_{p} \in\left[1, n_{b}\right]$;

- Set randomly another build, called destination build: $b_{d} \in\left[1, n_{b}\right]-\left\{b_{p}\right\}$;

- Exchange a discrete quantity $\varepsilon$ between selected builds, as the following equations indicate:

$$
n^{\text {new }}{ }_{i, b_{p}}=n_{i, b_{p}}-\varepsilon ; n^{n e w}{ }_{i, b_{d}}=n_{i, b_{d}}+\varepsilon \text {, with } \varepsilon \in\left[1, n_{i, b_{p}}\right]
$$

Once the above steps have been completed, the algorithm saves the value of OF with the attributes of the swap move in a TL and restarts the algorithm as previously introduced. These operations are synthetically called Schedule Screening. With the OF value, the following vector is recorded also with the chosen geometry, the starting and destination build and the exchange value between the two builds will selected and recorded in in the vector named attribute.

$$
\text { attributes }=\left[G_{i}, b_{p}, b_{d}, \varepsilon\right] .
$$

The proposed swap movement respects the production constraint because it is an exchange of a quantity $\varepsilon$ along a single order, so it is moved only a quantity from an addendum to another, having the total sum still the same; nevertheless, it has to respect the chamber volume constraint, so not all the swap moves are feasible and they require a check of feasibility before the OF is calculated (even if, as it will be

\begin{tabular}{|c|c|c|c|c|c|c|}
\hline \multirow{2}{*}{\multicolumn{2}{|c|}{$S$}} & \multicolumn{4}{|c|}{ Geometries } & \\
\hline & & 1 & 2 & 3 & 4 & \\
\hline \multirow{4}{*}{$\frac{\text { 을 }}{\bar{y}}$} & 1 & -7 & 0 & 4 & 1 & OF $=7.64$ \\
\hline & 2 & $\rightarrow 0$ & 7 & 0 & 1 & \\
\hline & 3 & 0 & 0 & 1 & 0 & \\
\hline & 4 & 0 & 1 & 0 & 4 & \\
\hline
\end{tabular}
explained later, some moves to avoid this inconvenience are possible).

Fig. 6. Initial Schedule

Table 4 presents an initial random schedule with an OF value of 7.64. When the proposed swap is applied to the selected order $(i=1)$ and to builds $\left(\right.$ build $\left._{1,2}\right)$, the OF value decreases to 7.08 moving a single part to the second build and checks that the move is compliant with the chamber volume available and the sum of the parts is always equal to demand for the i-th geometry. It is worth to note that the screening steps are contained in a while loop with a termination criterion characterised by a not improving subsequent iterations equal to 2 . As mentioned before, the proposed swap movement does not produce any violation on the demand constraint but it is not possible to say the same about the geometrical volume constraint. In fact, it could happen that swapping a quantity $\varepsilon$ between two builds implies an unacceptable condition in the destination build that is already full. This case is represented using another example of scheduling, presented in Fig. 7. In this case, the swap could seem not acceptable for the movement of the 
quantity of the fifth geometry in the green rectangle. This exchange could lead to a violation of the volume chamber constraint, which is $13437.50 \mathrm{~cm}^{3}$ in our example. Nevertheless, it is worth noting that in the overall schedule, some free volume is available. In fact, it is possible to move away some geometries from build \#2 to make feasible the initial swap movement proposed. For instance, some units of orders in the blue rectangle could be allocated in other free builds for the same geometries (for instance, in the yellow square builds) verifying the total quantity of the geometry.

\begin{tabular}{|c|c|c|c|c|c|c|c|c|c|}
\hline & \multirow{2}{*}{\multicolumn{2}{|c|}{$s$}} & \multicolumn{5}{|c|}{ Geometries } & \multirow{2}{*}{$V_{\text {used }}\left[\mathrm{cm}^{3}\right]$} & \multirow{2}{*}{$V_{\text {available }}\left[\mathrm{cm}^{3}\right]$} \\
\hline & & & 1 & 2 & 3 & 4 & 5 & & \\
\hline \multirow{6}{*}{ 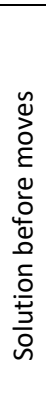 } & \multirow{6}{*}{$\frac{\overline{0}}{\frac{1}{3}}$} & 1 & 69 & 2 & 21 & 1 & 40 & 4329.09 & 9108.41 \\
\hline & & 2 & 4 & 24 & 30 & 1 & 133 & 13419.84 & 17.66 \\
\hline & & 3 & 3 & 23 & 55 & 8 & 127 & 13396.07 & 41.43 \\
\hline & & 4 & 64 & 85 & 91 & 274 & 62 & 13385.5 & 52 \\
\hline & & 5 & 9 & 55 & 111 & 16 & 115 & 13394.9 & 42.6 \\
\hline & & 6 & 1 & 11 & 92 & 0 & 123 & 13434.71 & 2.79 \\
\hline
\end{tabular}

Fig. 7. Volume management 1 of 2

This re-allocation, when the chamber volume constraint is violated, could be executed through an adaptation algorithm proposed here that automatically moves a fixed number of units to make an overflowing build feasible again. The process is reported in the following steps:

1. Compute extra-volume;

2. Compute the number of geometries necessary to reset extra volume and

3. Relocate each group of geometry in the following available builds.

As the last part of the algorithm has been explained, let us to refer to the following figure to represent the PTS proposed here (Fig. 8).

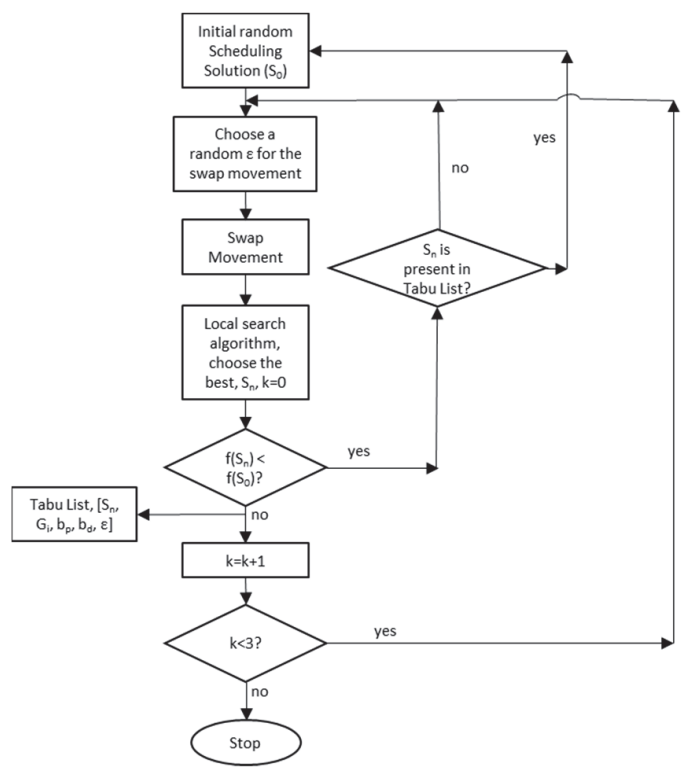

Fig. 8. The PTS proposed 


\subsection{Test case}

To better estimate the goodness of the proposed TS (Fig. 8) the test case presented in Fera et al. (2018) will be used. This was done to reduce the bias related to the test case building that could infer significant advantages to the new proposed method of solution. Therefore, the two heuristics tested will be the PTS here proposed and the genetic algorithm proposed in the 2018 paper. The comparison of the two heuristics will look at the following aspects:

- respect of due dates measured as the service level (i.e. the number of orders delivered compared to the ones of the demand vector),

- cost reduction, and

- elaboration time for each algorithm.

The Test case data (Table 1) are built to stress the scheduling problem faced. It is constituted by $30 \mathrm{PNs}$ that are very different from one another; all of them are parts with very low demand volumes.

Table 1

Test case data

\begin{tabular}{|c|c|c|c|c|c|c|c|c|}
\hline $\mathrm{PN}[\#]$ & $\mathrm{DD}[d a y]$ & $\mathrm{D}[$ part $]$ & $\mathrm{V}\left[\mathrm{cm}^{3}\right]$ & $\mathrm{h}[\mathrm{mm}]$ & $\rho\left[\mathrm{g} / \mathrm{cm}^{3}\right]$ & $\mathrm{T}_{\text {prep }}[$ hours $]$ & Penalty $[\% / d a y]$ & $\mathrm{s}_{\max }\left[\mathrm{cm}^{2}\right]$ \\
\hline 1 & 120 & 7 & 146 & 50.55 & 8 & 1 & 1 & 82.1 \\
\hline 2 & 60 & 5 & 52.87 & 85 & 8 & 1 & 2 & 120 \\
\hline 3 & 180 & 10 & 108,9 & 62.5 & 8 & 1 & 2 & 344.22 \\
\hline 4 & 120 & 7 & 64.17 & 37.73 & 8 & 1 & 1.5 & 21.00 \\
\hline 5 & 120 & 5 & 200.8 & 183.4 & 8 & 0.5 & 3 & 208.08 \\
\hline 6 & 90 & 5 & 66.94 & 56.02 & 8 & 1 & 1.5 & 178.72 \\
\hline 7 & 120 & 5 & 90.15 & 95 & 8 & 0.5 & 2 & 57.04 \\
\hline 8 & 120 & 8 & 188.2 & 162.5 & 8 & 1 & 2 & 104.56 \\
\hline 9 & 150 & 9 & 33.65 & 32.29 & 8 & 1 & 1.5 & 97.12 \\
\hline 10 & 180 & 9 & 290.2 & 186.6 & 8 & 1 & 1.5 & 112.28 \\
\hline 11 & 60 & 5 & 62 & 150 & 8 & 0.5 & 1 & 176.71 \\
\hline 12 & 180 & 10 & 6 & 73 & 8 & 1 & 2 & 41.85 \\
\hline 13 & 180 & 10 & 9 & 65 & 8 & 0.4 & 1 & 33.18 \\
\hline 14 & 90 & 8 & 56 & 115 & 8 & 0.6 & 2 & 103.869 \\
\hline 15 & 120 & 5 & 17 & 100 & 8 & 0.4 & 1 & 213.82 \\
\hline 16 & 150 & 8 & 44 & 165 & 8 & 0.4 & 3 & 78.54 \\
\hline 17 & 120 & 7 & 4.87 & 100 & 8 & 0.1 & 1 & 3.14 \\
\hline 18 & 60 & 5 & 2,9 & 22 & 8 & 0.2 & 1 & 38.48 \\
\hline 19 & 180 & 10 & 112 & 70 & 8 & 0.4 & 1 & 116.90 \\
\hline 20 & 90 & 5 & 150 & 122 & 8 & 0.4 & 2 & 201.06 \\
\hline 21 & 150 & 8 & 375 & 160 & 8 & 0.7 & 1 & 4.91 \\
\hline 22 & 60 & 5 & 17.5 & 25 & 8 & 0.1 & 1 & 28.27 \\
\hline 23 & 90 & 6 & 36 & 60 & 8 & 0.2 & 1 & 12.57 \\
\hline 24 & 120 & 7 & 13.4 & 40 & 8 & 0.2 & 1 & 10.18 \\
\hline 25 & 90 & 5 & 22.6 & 36 & 8 & 0.3 & 2 & 9.62 \\
\hline 26 & 120 & 7 & 7 & 35 & 8 & 0.4 & 2 & 12.57 \\
\hline 27 & 60 & 4 & 11 & 40 & 8 & 0.4 & 1 & 10.18 \\
\hline 28 & 90 & 5 & 4 & 45 & 8 & 0.4 & 2 & 9.62 \\
\hline 29 & 120 & 7 & 15 & 50 & 8 & 0.4 & 2 & 19.63 \\
\hline 30 & 180 & 10 & 0.569 & 20 & 8 & 0.4 & 2 & 3.14 \\
\hline
\end{tabular}

The deliveries have to be performed from the 60th to the 180th day, i.e. the demand covers a time span of 6 months. The build chamber volume is $V_{\max }=13437.50 \mathrm{~cm}^{3}$. The solution algorithms run for 250 repetitions, the experiments revealed no particular variances of the results; so, it is worth to note that the results are the mean values of the parameters before introduced calculated on the population of the 250 experiments. The standard deviation of the experimental results was almost of $6.6 \%$, measured as the mean of the ratio of the standard deviation of each evaluation parameter and the mean value. The simulation runs were performed on a calculation machine with Intel Core i7 ${ }^{-7700 H Q, C P U}$ of $2.80 \mathrm{GHz}$ and $16 \mathrm{~GB}$ of $R A M$. All algorithms are coded in Matlab ${ }^{\circledR} 7$ R2015a. It is worth noting that the CPU frequency is comparable with the one used for the experiment by Fera et al. in 2018; therefore, the results can be compared. As underlined in the paper whose results are compared with the current results, a value of the $\mathrm{k}$ parameter $0<k \leq 1$ capable of modifying the maximum chamber volume is chosen: 


$$
V_{\text {available }}=k \times V_{\text {max }} \text {. }
$$

This simple algorithm generates a first random vector of integers, by randi() Matlab ${ }^{\circledR}$ function; after this, the algorithm verifies production and volume constraints. If the build is feasible and partially empty, an Optimisation Module starts to fill it with the PNs with a sooner due date. The random build generation, as well as the $k$ parameter, contribute to diversification of solutions. A possible initial feasible population for the test case data is computed using the previous said Matlab module; however, due to less space, it is not shown.

\subsection{The PTS and PGA results}

Before to start with the analysis of the results; note that the initial values of the evaluation parameters are similar among the two methods used, so it is worth to investigate also the percentage improvement given by the use of the two heuristics. PTS needs only one schedule as input, so we selected one feasible in the initial set, with the least OF value $F_{S}=29.20$. As explained before, the PTS procedure needs to define a proper value for $\varepsilon$. After the result given by the selection of the value $\varepsilon=-1$ are presented; we can appreciate a OF reduction of $82.00 \%$; running time for PTS is 1.32 minutes. The genetic algorithm (GA) used was the one proposed in 2018 (PGA) gives good solutions, with sensible reduction of OF, which are worse than those of PTS. In all the 250 experiments, iteration numbers for the PGA are greater than 1000 in almost all experiments and less only in one case (i.e. the tenth one, with a total number of iteration equal to 478, which presents the worst result among trials). For the genetic algorithm the OF improvement is higher than $75 \%$, whereas the cost of production decreased by $8.12 \%$ and the LS increased by $32.12 \%$.

Table 2

The results of Genetic Algorithm and TS

\begin{tabular}{|c|c|c|c|c|c|}
\hline & & & Initial & Final & $\Delta[\%]$ \\
\hline \multirow[t]{3}{*}{ Genetic } & OF & {$[-]$} & 29.20 & 7.01 & -75.9 \\
\hline & CP & {$[\mathrm{k} €]$} & 163.30 & 150.06 & -8.12 \\
\hline & LS & {$[\%]$} & 49.12 & 65.00 & +32.12 \\
\hline \multirow[t]{3}{*}{ Tabu Search } & OF & {$[-]$} & 29.20 & 4.89 & -82.00 \\
\hline & CP & {$[\mathrm{k} \epsilon]$} & 165.30 & 145.57 & -11.94 \\
\hline & LS & {$[\%]$} & 46.67 & 80.00 & +71.43 \\
\hline
\end{tabular}

The overall results for the genetic algorithm are worse than those for PTS ones, but the remark of PGA is to make sure the running time is $0.20 \mathrm{~min}$, i.e. about $12 \mathrm{~s}$. The repetitions of the experimental cases and the low value of the standard deviation of the outputs of simulation lead us to affirm that the PTS performances are better than the genetic algorithm, even if the running time of execution of the two heuristics is sensibly different with a clear advantage in using the genetic algorithm. The running time of the calculation procedures could be an important issue when the problem size increases and goes up in umber of part numbers and variable examined and calculated by the iterative procedures.

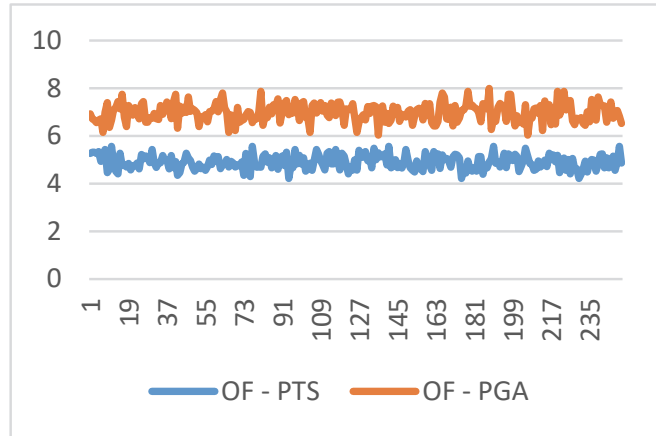

Fig. 9. Comparison of the 250 experimental values for the OF using PTS and PGA

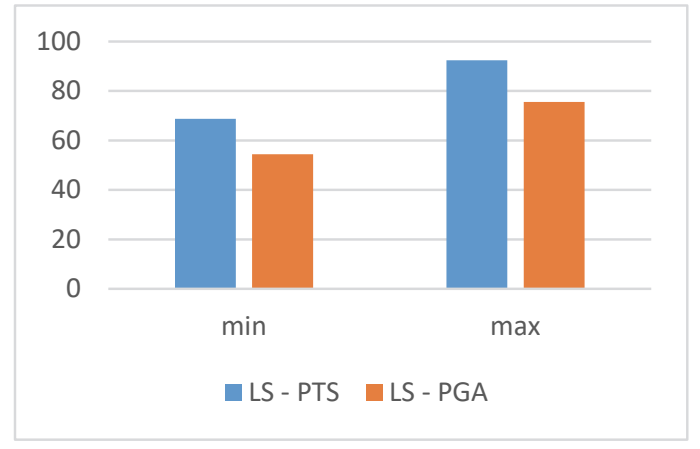

Fig. 10. Comparison of the minimum and maximum values of the service level for the 250 experiments 
The following presents the comparison for single evaluation parameter. Let us start with the value of the objective function. In Fig. 9, the orange points represent the values of the objective function using the genetic algorithm and the blue points are, instead, the results of the model using the tabu search, as it is possible to see the results of PTS are always less than the ones using the PGA. The shapes of the other evaluation parameters are more or less similar to the ones showed in Fig. 9. It is worth to note, instead, that the service level offered by each method present an intersection of the areas of minimum and maximum for all the experiments as shown in Fig. 10. Lastly, below (in Table 3) it is reported the standard deviation measured as percentage of the mean value for the specific evaluation parameter analysed. As it is possible to see the deviation is low for all the parameters used.

Table 3

Standard deviation of the results produced by the solution method for a sample of 250 experiments

\begin{tabular}{cccccccccc}
\hline & \multicolumn{4}{c}{ PTS } & \multicolumn{4}{c}{ PGA } \\
\cline { 2 - 9 } & OF - PTS & CP & LS & Time execution & OF - PGA & CP & LS & Time execution \\
\hline Standard Deviation & $5,62 \%$ & $6,48 \%$ & $6,00 \%$ & $7,63 \%$ & $5,57 \%$ & $5,58 \%$ & $6,51 \%$ & $6,98 \%$ & 0 \\
\hline
\end{tabular}

\section{Conclusions and discussion of the results}

Herein, an updated version of a recently presented optimisation model has been illustrated for the operations management optimisation using a single machine with the AM technology. Moreover, a new heuristic to face this problem specifically applied to the AM technology was presented. The updated mathematical model (that is a NP-HARD problem) was solved adapting a traditional heuristic to respect the AM technological characteristics, i.e. the TS algorithm. This algorithm was applied to the same test cases used by Fera et al. (2018), to guarantee the bias absence due to the test case building. The test case used is the one in which a traditional manufacturing production system receives orders that generally have difficulties to be respected since they are low in volumes orders and with high geometrical variability. At the end of this paper, it is possible to compare the results of these two heuristics, i.e. the PGA and the PTS. It is possible to see a significant advantage to the PTS results in terms of operations management performances. The only point in favour of the PGA is the running time, which is $12 \mathrm{~s}$ instead of $92 \mathrm{~s}$ for the PTS. Therefore, it is possible to say that in terms of efficiency, the PGA seems to be a better solver than the PTS, even if the operative results are in favour of the PTS. Nevertheless, in case the number of orders of different part numbers grows significatively the cited efficiency of genetic algorithm could be a winning key of analysis, neglecting a better result in terms of key performance indicators (KPI) for operations management. In fact, the PTS is better than the PGA for all the three evaluation parameters (i.e. the value of the OF, the value of production costs and the service level percentage), whereas it is less performing than the PGA in terms of the running time. In future, other possible heuristics could be applied to the specific management problem here presented and possible improvement of both the KPI for the operations management and for the running time of calculation execution could be individuated.

\section{References}

Atzeni, E., \& Salmi, A. (2012). Economics of additive manufacturing for end-usable metal parts. The International Journal of Advanced Manufacturing Technology, 62(9-12), 1147-1155.

Chergui, A., Hadj-Hamou, K., \& Vignat, F. (2018). Production scheduling and nesting in additive manufacturing. Computers \& Industrial Engineering, 126, 292-301.

Costabile, G., Fera, M., Fruggiero, F., Lambiase, A., \& Pham, D. (2017). Cost models of additive manufacturing: A literature review. International Journal of Industrial Engineering Computations, 8(2), 263-283.

Dilberoglu, U. M., Gharehpapagh, B., Yaman, U., \& Dolen, M. (2017). The role of additive manufacturing in the era of industry 4.0. Procedia Manufacturing, 11, 545-554.

Fera, M., Fruggiero, F., Lambiase, A., \& Macchiaroli, R. (2016). State of the art of additive manufacturing: Review for tolerances, mechanical resistance and production costs. Cogent Engineering, 3(1), 1261503. 
Fera, M., Macchiaroli, R., Fruggiero, F., \& Lambiase, A. (2018). A new perspective for production process analysis using additive manufacturing - complexity vs production volume. The International Journal of Advanced Manufacturing Technology, 95(1-4), 673-685.

Fruggiero, F., Riemma, S., Ouazene, Y., Macchiaroli, R., \& Guglielmi, V. (2016). Incorporating the human factor within manufacturing dynamics. IFAC-PapersOnLine, 49(12), 1691-1696.

Fera, M., Costabile, G., Fruggiero, F., Lambiase, A., \& Pham, D. T. (2017). A new mixed production cost allocation model for additive manufacturing (MiProCAMAM). International Journal of Advanced Manufacturing Technology, 92(9-12), 42754291.

Fera, M., Fruggiero, F., Macchiaroli R., Lambiase, A., Todisco, V. (2018). A modified genetic algorithm for time and cost optimization of an additive manufacturing single-machine scheduling. International Journal of Industrial Engineering Computations, 9(1), pp. 1-16.

Glover, F., \& Laguna, M. (1997a). General purpose heuristics for integer programming-Part I. Journal of Heuristics, 2(4), 343-358.

Glover, F., \& Laguna, M. (1997b). General purpose heuristics for integer programming — part II. Journal of Heuristics, 3(2), 161-179.

Jin, Y., Du, J., \& He, Y. (2017). Optimization of process planning for reducing material consumption in additive manufacturing. Journal of Manufacturing Systems, 44, 65-78.

Khajavi, S. H., Partanen, J., \& Holmström, J. (2014). Additive manufacturing in the spare parts supply chain. Computers in Industry, 65(1), 50-63.

Kucukkoc, I. (2019). MILP models to minimise makespan in additive manufacturing machine scheduling problems. Computers \& Operations Research, 105, 58-67.

Li, Q., Kucukkoc, I., \& Zhang, D. Z. (2017). Production planning in additive manufacturing and 3D printing. Computers \& Operations Research, 83, 157-172.

Newman, S. T., Zhu, Z., Dhokia, V., \& Shokrani, A. (2015). Process planning for additive and subtractive manufacturing technologies. CIRP Annals-Manufacturing Technology, 64(1), 467-470.

Pour, M. A., Zanardini, M., Bacchetti, A., \& Zanoni, S. (2016). Additive manufacturing impacts on productions and logistics systems. IFAC-Papers On Line, 49(12), 1679-1684.

Ransikarbum, K., Ha, S., Ma, J., \& Kim, N. (2017). Multi-objective optimization analysis for part-to-Printer assignment in a network of 3D fused deposition modeling. Journal of Manufacturing Systems, 43, 35-46.

Ren, L., Sparks, T., Ruan, J., \& Liou, F. (2008). Process planning strategies for solid freeform fabrication of metal parts. Journal of Manufacturing Systems, 27(4), 158-165.

Rickenbacher, L., Spierings, A., \& Wegener, K. (2013b) An integrated cost-model for selective laser melting (SLM). Rapid Prototyp Journal, 19(3), 208-214

Ruffo, M., \& Hague, R. (2007). Cost estimation for rapid manufacturing'simultaneous production of mixed components using laser sintering. Proceedings of the Institution of Mechanical Engineers, Part B: Journal of Engineering Manufacture, 221(11), 1585-1591.

Strong, D., Kay, M., Conner, B., Wakefield, T., \& Manogharan, G. (2018). Hybrid manufacturing-integrating traditional manufacturers with additive manufacturing supply chain. Additive Manufacturing, 21, 159-173.

Verboeket, V., \& Krikke, H. (2019). The disruptive impact of additive manufacturing on supply chains: A literature study, conceptual framework and research agenda. Computers in Industry, 111, 91-107.

Witherell, P., Lu, Y., \& Jones, A. (2017). Additive manufacturing: A trans-disciplinary experience. In Transdisciplinary Perspectives on Complex Systems (pp. 145-175). Springer International Publishing.

Zhang, Y., Gupta, R. K., \& Bernard, A. (2016). Two-dimensional placement optimization for multi-parts production in additive manufacturing. Robotics and Computer-Integrated Manufacturing, 38, 102-117.

Zhu, Z., Dhokia, V., \& Newman, S. T. (2017). A novel decision-making logic for hybrid manufacture of prismatic components based on existing parts. Journal of Intelligent Manufacturing, 28(1), 131-148.

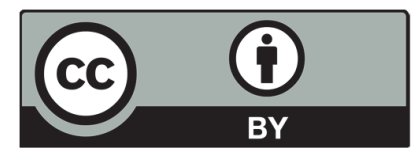

C 2020 by the authors; licensee Growing Science, Canada. This is an open access article distributed under the terms and conditions of the Creative Commons Attribution (CCBY) license (http://creativecommons.org/licenses/by/4.0/). 